\title{
Two neutrino double-beta decay and effective axial-vector coupling constant
}

\author{
Fedor Šimkovic ${ }^{1,3.4, *}$, Rastislav Dvornický1 ${ }^{1,2, * *}$, and Dušan Štefánik ${ }^{1, * * *}$ \\ ${ }^{1}$ Comenius University, Mlynská dolina F1, SK-842 48 Bratislava, Slovakia \\ ${ }^{2}$ Dzhelepov Laboratory of Nuclear Problems, JINR, 141980 Dubna, Russia \\ ${ }^{3}$ Bogoliubov Laboratory of Theoretical Physics, JINR, 141980 Dubna, Russia \\ ${ }^{4}$ Czech Technical University in Prague, 128-00 Prague, Czech Republic
}

\begin{abstract}
The theoretical and experimental study of the two-neutrino double-beta decay ( $2 v \beta \beta$-decay) is of crucial importance for reliable calculation of matrix elements governing the neutrinoless double-beta decay ( $0 v \beta \beta$-decay). That will allow to determine masses of neutrinos once the $0 v \beta \beta$-decay, which occurs if the neutrino is a massive Majorana particle and the total lepton number is not conserved, will be observed. Experiments studying the $2 v \beta \beta$-decay are currently approaching a qualitatively new level, where high-precision measurements are performed not only for half-lives but for all other observables of the process. In this context an improved formula for the $2 v \beta \beta$-decay is presented. Further, a novel approach for determining the effective axial-vector coupling constant is proposed.
\end{abstract}

\section{Introduction}

The observation of the neutrinoless double beta decay process $(0 v \beta \beta$-decay) [1],

$$
(A, Z) \rightarrow(A, Z+2)+2 e^{-},
$$

will not only demonstrate the Majorana nature of neutrinos but it will also provide a measurement of the effective Majorana neutrino mass,

$$
m_{\beta \beta}=U_{e 1}^{2} m_{1}+U_{e 2}^{2} m_{2}+U_{e 3}^{2} m_{3} .
$$

Here, $U_{e i}$ and $m_{i}(\mathrm{i}=1,2$, and 3$)$ are elements of the Pontecorvo-Maki-Nakagawa-Sakata neutrino mixing matrix and masses of neutrinos, respectively. The inverse value of the $0 v \beta \beta$-decay half-life is commonly written as

$$
\left(T_{1 / 2}^{0 v \beta \beta}\right)^{-1}=\left|\frac{m_{\beta \beta}}{m_{e}}\right|^{2}\left(g_{A}^{\mathrm{eff}}\right)^{4}\left|M_{G T}^{0 v}\right|^{2} G^{0 v},
$$

where $M^{0 v}, g_{A}^{\text {eff }}$ and $G^{0 v}$ are the nuclear matrix element, effective axial-vector coupling constant, and phase-space factor, respectively. The calculation of $M^{0 v}$ by using nuclear structure theory [2] and determination of $g_{A}^{\text {eff }}$ [3] are crucial for the interpretation of the $0 v \beta \beta$-decay.

Understanding the two-neutrino double-beta decay

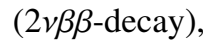

$$
(A, Z) \rightarrow(A, Z+2)+2 e^{-}+2 \bar{v}_{e},
$$

which involves the emission of two electrons and two antineutrinos, remains of major importance for reliable calculation of the $0 v \beta \beta$-decay nuclear matrix elements.

\footnotetext{
*e-mail: simkovic@ fmph.uniba.sk

**e-mail: rastonator@gmail.com

***e-mail: dus.stefanik@gmail.com
}

The $2 v \beta \beta$-decay has been a subject of experimental research for more than 60 years. It has been detected for 12 different nuclei for transition to the ground state and in two cases also to transition to $0^{+}$excited state of the daughter nucleus [4]. This rare process is one of the major sources of background in running and planned experiments looking for a signal of the $0 v \beta \beta$-decay.

In this contribution a more accurate expression for the $2 v \beta \beta$-decay half-life is presented. In addition, a novel approach for determining the effective value of the axialvector coupling constant $g_{A}^{\mathrm{eff}}$ is suggested.

\section{The improved $2 v \beta \beta$-decay rate}

The inverse half-life of the $2 v \beta \beta$-decay transition to the $0^{+}$ ground state of the final nucleus takes the form [5]

$$
\left[T_{1 / 2}^{2 v \beta \beta}\right]^{-1}=\frac{m_{e}}{8 \pi^{7} \ln 2}\left(G_{\beta} m_{e}^{2}\right)^{4}\left(g_{A}^{\mathrm{eff}}\right)^{4} I^{2 v},
$$

where $G_{\beta}=G_{F} \cos \theta_{C}\left(G_{F}\right.$ is Fermi constant and $\theta_{C}$ is the Cabbibo angle), $m_{e}$ is the mass of electron and

$$
\begin{aligned}
I^{2 v}=\frac{1}{m_{e}^{11}} & \int_{m_{e}}^{E_{i}-E_{f}-m_{e}} F_{0}\left(Z_{f}, E_{e_{1}}\right) p_{e_{1}} E_{e_{1}} d E_{e_{1}} \times \\
& \int_{m_{e}}^{E_{i}-E_{f}-E_{e_{1}}} F_{0}\left(Z_{f}, E_{e_{2}}\right) p_{e_{2}} E_{e_{2}} d E_{e_{2}} \times \\
& \int_{0}^{E_{i}-E_{f}-E_{e_{1}}-E_{e_{2}}} E_{v_{1}}^{2} E_{v_{2}}^{2} \mathcal{A}^{2 v} d E_{v_{1}} .
\end{aligned}
$$

Here, $E_{v_{2}}=E_{i}-E_{f}-E_{e_{1}}-E_{e_{2}}-E_{v_{1}}$ due to energy conservation. $E_{i}, E_{f}, E_{e_{i}}\left(E_{e_{i}}=\sqrt{p_{e_{i}}^{2}+m_{e}^{2}}\right)$, and $E_{v_{i}}(i=1,2)$ are the energies of initial and final nuclei, electrons and antineutrinos, correspondingly. $F_{0}\left(Z_{f}, E_{e_{i}}\right)$ denotes relativistic Fermi function and $Z_{f}=Z+2$. $\mathcal{A}^{2 v}$ consists of products 


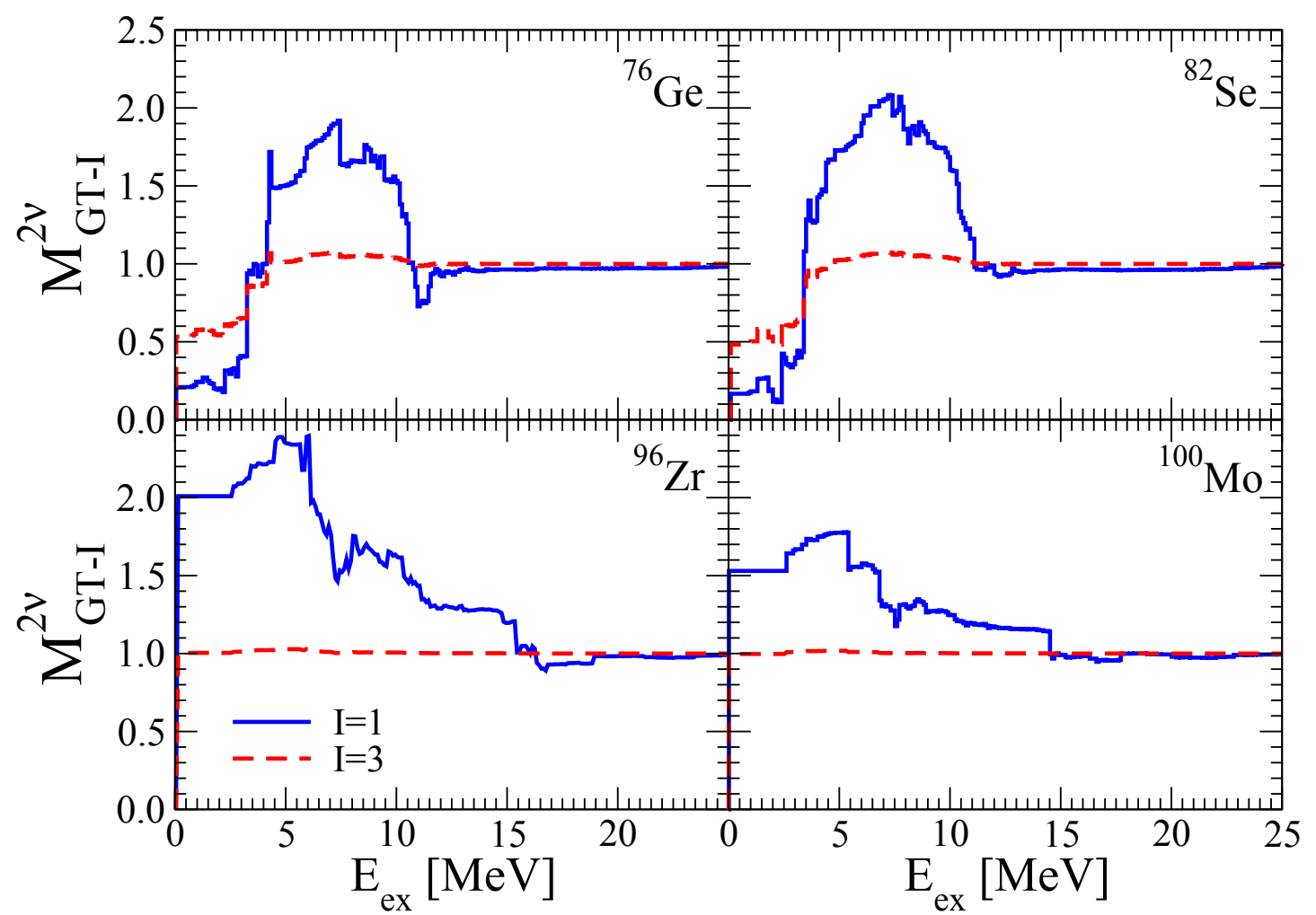

Figure 1. (Color online) Running sum of the matrix elements $M_{G T-1}^{2 v}$ and $M_{G T-3}^{2 v}$ (normalized to unity) as a function of the excitation energy $E_{e x}$ counted from the ground state of intermediate nucleus for the $2 v \beta \beta$-decay of ${ }^{76} \mathrm{Ge},{ }^{82} \mathrm{Se},{ }^{96} \mathrm{Zr}$, and ${ }^{100} \mathrm{Mo}$. Calculation was performed within the proton-neutron QRPA with isospin restoration [8]. Results are obtained with Argonne V18 potential and for unquenched axial vector coupling constant $g_{A}=1.269$.

of the Gamow-Teller nuclear matrix elements (we neglect the contribution from the double Fermi transitions to the $2 v \beta \beta$-decay rate), which depend on lepton energies

$$
\mathcal{A}^{2 v}=\left[\frac{1}{4}\left|M_{G T}^{K}+M_{G T}^{L}\right|^{2}+\frac{1}{12}\left|M_{G T}^{K}-M_{G T}^{L}\right|^{2}\right],
$$

where

$$
M_{G T}^{K, L}=m_{e} \sum_{n} M_{n} \frac{E_{n}-\left(E_{i}+E_{f}\right) / 2}{\left[E_{n}-\left(E_{i}+E_{f}\right) / 2\right]^{2}-\varepsilon_{K, L}^{2}},
$$

with

$$
M_{n}=\left\langle 0_{f}^{+}\left\|\sum_{m} \tau_{m}^{-} \sigma_{m}\right\| 1_{n}^{+}\right\rangle\left\langle 1_{n}^{+}\left\|\sum_{m} \tau_{m}^{-} \sigma_{m}\right\| 0_{i}^{+}\right\rangle .
$$

Here, $\left|0_{i}^{+}\right\rangle\left(\left|0_{f}^{+}\right\rangle\right)$is ground state of the initial (final) eveneven nuclei with energy $E_{i}\left(E_{f}\right)$, and $\mid 1_{n}^{+}>$are the states in the intermediate odd-odd nucleus with energies $E_{n}$. The lepton energies enter in the factors

$$
\begin{aligned}
\varepsilon_{K} & =\left(E_{e_{2}}+E_{v_{2}}-E_{e_{1}}-E_{v_{1}}\right) / 2, \\
\varepsilon_{L} & =\left(E_{e_{1}}+E_{v_{2}}-E_{e_{2}}-E_{v_{1}}\right) / 2 .
\end{aligned}
$$

The maximal value of $\left|\varepsilon_{K}\right|$ and $\left|\varepsilon_{L}\right|$ is the $Q$ value of the process $\left(\varepsilon_{K} \in(-Q, Q)\right)$. For $2 v \beta \beta$-decay with energetically forbidden transition to intermediate nucleus $\left(E_{n}-\right.$ $\left.E_{i}>-m_{e}\right)$ the quantity $2 E_{n}-E_{i}-E_{f}=Q+2 m_{e}+2\left(E_{n}-E_{i}\right)$ is always larger than the $Q$ value.

The standard treatment of the $2 v \beta \beta$-decay [1]: The calculation of the $2 v \beta \beta$-decay probability is usually simplified by neglecting the factors $\varepsilon_{K, L}$ in energy denominators, what allows a separate calculation of the phase space factor and nuclear matrix element. Then, the inverse half-life of the $2 v \beta \beta$-decay can be written as

$$
\left(T_{1 / 2}^{2 v \beta \beta}\right)^{-1}=\left(g_{A}^{\mathrm{eff}}\right)^{4}\left|M_{G T}^{2 v}\right|^{2} G^{2 v}
$$

where $G^{2 v}$ is the phase-space factor [6]. The double Gamow-Teller (GT) matrix element is expressed in terms of sequential single $\beta$-decay Gamow-Teller transitions through virtual $1^{+}$intermediate states.

$$
M_{G T}^{2 v}=m_{e} \sum_{n} \frac{M_{n}}{E_{n}-\left(E_{i}+E_{f}\right) / 2} .
$$

An improved description of the $2 v \beta \beta$-decay [7]: A more accurate expression for the $2 v \beta \beta$-decay rate we get 
Table 1. Nuclear matrix elements $M_{G T-1}^{2 v}$ and $M_{G T-3}^{2 v}$ for $2 v \beta \beta$-decay of ${ }^{76} \mathrm{Ge},{ }^{82} \mathrm{Se},{ }^{96} \mathrm{Zr}$, and ${ }^{76} \mathrm{Mo}$ calculated within the QRPA with partial restoration of isospin symmetry [8]. For a given value of effective axial-vector coupling constant $g_{A}^{\text {eff }}$ the strength of isoscalar interaction was adjusted to the measured $2 v \beta \beta$-decay half-life $T_{1 / 2}^{2 v-e x p}[4]$ and the strength of isovector interaction by the fulfillment of

the requirement that the $2 v \beta \beta$ Fermi matrix vanishes [8]. $P_{0}^{2 v}$ and $P_{2}^{2 v}$ are the leading order and first order Taylor expansion contributions to $2 v \beta \beta$-decay width relative to the full width. $G_{0}^{2 v}$ and $G_{2}^{2 v}$ are phase-space integrals entering the expression for the $2 v \beta \beta$-decay rate in Eqs. (13) and (14), which were calculated with help of the exact Dirac wave functions with finite nuclear size and electron screening of the electron [6].

\begin{tabular}{cccccccccc}
\hline nucl. & $g_{A}^{\text {eff }}$ & $M_{G T-1}^{2 v}$ & $M_{G T-3}^{2 v}$ & $\xi_{13}^{2 v}$ & $P_{0}^{2 v}$ & $P_{2}^{2 v}$ & $G_{0}^{2 v}\left[\mathrm{yr}^{-1}\right]$ & $G_{2}^{2 v}\left[\mathrm{yr}^{-1}\right]$ & $T_{1 / 2}^{2 v-e x p}[\mathrm{yr}]$ \\
\hline${ }^{76} \mathrm{Ge}$ & 0.800 & 0.175 & 0.0214 & 0.1220 & 0.975 & 0.025 & $4.81610^{-20}$ & $1.01510^{-20}$ & $1.6510^{21}$ \\
& 1.000 & 0.111 & 0.0133 & 0.1204 & 0.975 & 0.025 & & & \\
& 1.269 & 0.0689 & 0.00716 & 0.1040 & 0.979 & 0.021 & & & \\
${ }^{82} \mathrm{Se}$ & 0.800 & 0.124 & 0.0216 & 0.1745 & 0.928 & 0.072 & $1.59110^{-18}$ & $7.03710^{-19}$ & $0.9210^{20}$ \\
& 1.000 & 0.0795 & 0.0129 & 0.1620 & 0.933 & 0.067 & & & \\
& 1.269 & 0.0498 & 0.00643 & 0.1290 & 0.945 & 0.055 & & & \\
${ }^{96} \mathrm{Zr}$ & 0.800 & 0.1146 & 0.0348 & 0.3036 & 0.857 & 0.143 & $6.79610^{-18}$ & $3.74510^{-18}$ & $2.310^{19}$ \\
& 1.000 & 0.0718 & 0.273 & 0.3800 & 0.827 & 0.173 & & & \\
${ }^{100} \mathrm{Mo}$ & 1.269 & 0.0431 & 0.0220 & 0.5101 & 0.781 & 0.219 & & & \\
& 0.800 & 0.292 & 0.123 & 0.4230 & 0.838 & 0.162 & $3.30310^{-18}$ & $1.50910^{-18}$ & $7.110^{18}$ \\
& 1.000 & 0.184 & 0.0876 & 0.4752 & 0.822 & 0.178 & & & \\
& 1.269 & 0.112 & 0.0633 & 0.5646 & 0.795 & 0.205 & & & \\
\hline
\end{tabular}

by performing the Taylor expansion in matrix elements $M_{G T}^{K, L}$ over the ratio $\varepsilon_{K, L} /\left(E_{n}-\left(E_{i}+E_{f}\right) / 2\right)$ [7]. By limiting our consideration to the second power in $\varepsilon$ we end up with

$$
\left[T_{1 / 2}^{2 v \beta \beta}\right]^{-1} \simeq \frac{\Gamma_{0}^{2 v}+\Gamma_{2}^{2 v}}{\ln (2)}
$$

where partial contributions to the full $2 v \beta \beta$-decay width $\Gamma^{2 v}$ associated with the leading $\Gamma_{0}^{2 v}$, next to leading $\Gamma_{2}^{2 v}$ order in Taylor expansion are given by

$$
\begin{aligned}
\frac{\Gamma_{0}^{2 v}}{\ln (2)} & =\left(g_{A}^{\mathrm{eff}}\right)^{4}\left|M_{G T-1}^{2 v}\right|^{2} G_{0}^{2 v}, \\
\frac{\Gamma_{2}^{2 v}}{\ln (2)} & =\left(g_{A}^{\mathrm{eff}}\right)^{4} \mathfrak{R}\left\{M_{G T-1}^{2 v} M_{G T-3}^{2 v}\right\} G_{2}^{2 v} .
\end{aligned}
$$

Nuclear matrix elements are defined as

$$
\begin{aligned}
& M_{G T-1}^{2 v}=\sum_{n} M_{n} \frac{m_{e}}{\left(E_{n}-\left(E_{i}+E_{f}\right) / 2\right)} \equiv M_{G T}^{2 v}, \\
& M_{G T-3}^{2 v}=\sum_{n} M_{n} \frac{4 m_{e}^{3}}{\left(E_{n}-\left(E_{i}+E_{f}\right) / 2\right)^{3}} .
\end{aligned}
$$

The phase-space factors are given by

$$
\begin{aligned}
G_{N}^{2 v}=\frac{c_{2 v}}{m_{e}^{11}} & \int_{m_{e}}^{E_{i}-E_{f}-m_{e}} F_{0}\left(Z_{f}, E_{e_{1}}\right) p_{e_{1}} E_{e_{1}} d E_{e_{1}} \times \\
& \int_{m_{e}}^{E_{i}-E_{f}-E_{e_{1}}} F_{0}\left(Z_{f}, E_{e_{2}}\right) p_{e_{2}} E_{e_{2}} d E_{e_{2}} \times \\
& \int_{0}^{E_{i}-E_{f}-E_{e_{1}}-E_{e_{2}}} E_{v_{1}}^{2} E_{v_{2}}^{2} \mathcal{A}_{N}^{2 v} d E_{v_{1}}
\end{aligned}
$$

with $\mathrm{N}=0,2 . \quad c_{2 v}=m_{e}\left(G_{\beta} m_{e}^{2}\right)^{4} /\left(8 \pi^{7} \ln 2\right), \mathcal{A}_{0}^{2 v}=1$ and $\mathcal{A}_{2}^{2 v}=\left(\varepsilon_{K}^{2}+\varepsilon_{L}^{2}\right) /\left(2 m_{e}\right)^{2}$.

\section{A novel possibility to determine effective axial-vector coupling constant}

The effective axial-vector coupling constant $g_{A}^{\text {eff }}$ enters in the fourth power in both the $0 v \beta \beta$ - and $2 v \beta \beta$-decay rates and is a major concern of experiments looking for a signal of the $0 v \beta \beta$-decay as its value might be strongly suppressed when compared to $g_{A}=1.269$ at nucleon level. In Ref. [9], it was found $\left(g_{A}^{\text {eff }}\right)^{4}=0.30$ and 0.50 for the $2 v \beta \beta$-decay of ${ }^{100} \mathrm{Mo}$ and ${ }^{116} \mathrm{Cd}$, respectively. By performing calculation within the QRPA $g_{A}^{\text {eff }}$ was treated as a completely free parameter alongside $g_{p p}$, which is used to renormalize particle-particle interaction of the nuclear Hamiltonian. A least-squares fit of $g_{A}^{\text {eff }}$ and $g_{p p}$ to the $\beta$ decay rate and EC rate of the $1^{+}$ground state in the intermediate nuclei involved in the double-beta decay in addition to the $2 v \beta \beta$-decay rate of the initial nuclei, led to $g_{A}^{\text {eff }}$ of about $0.7\left({ }^{100} \mathrm{Mo}\right)$ and $0.8\left({ }^{116} \mathrm{Cd}\right)$. A significantly stronger quenching of the axial-vector coupling constant, namely $\left(g_{A}^{\text {eff }}\right)^{4} \simeq\left(1.269 A^{-0.18}\right)^{4}=0.063$, was found within the Interacting Boson Model [10]. It has been determined by the theoretical prediction for the $2 v \beta \beta$-decay half-lives, which were based on the closure approximation calculated nuclear matrix element $M_{G T}^{2 v}$, with the measured half-lives.

The value of effective axial-vector coupling constant $g_{A}^{\text {eff }}$ can be determined from the measured $2 v \beta \beta$-decay half-life (see Eqs. (13) and (14)) once nuclear matrix elements $M_{G T-1}^{2 v}$ and $M_{G T-3}^{2 v}$ are evaluated by using nuclear structure theory. Unfortunately, none of modern nuclear structure methods allows a reliable calculation of the matrix element $M_{G T-1}^{2 v}$, which is associated with a dominant contribution to the $2 v \beta \beta$-decay rate. An open question is whether contributions through the higher lying states of the intermediate nucleus play an important role in evaluation of $M_{G T-1}^{2 v}$. In the case of matrix element $M_{G T-3}^{2 v}$, which 


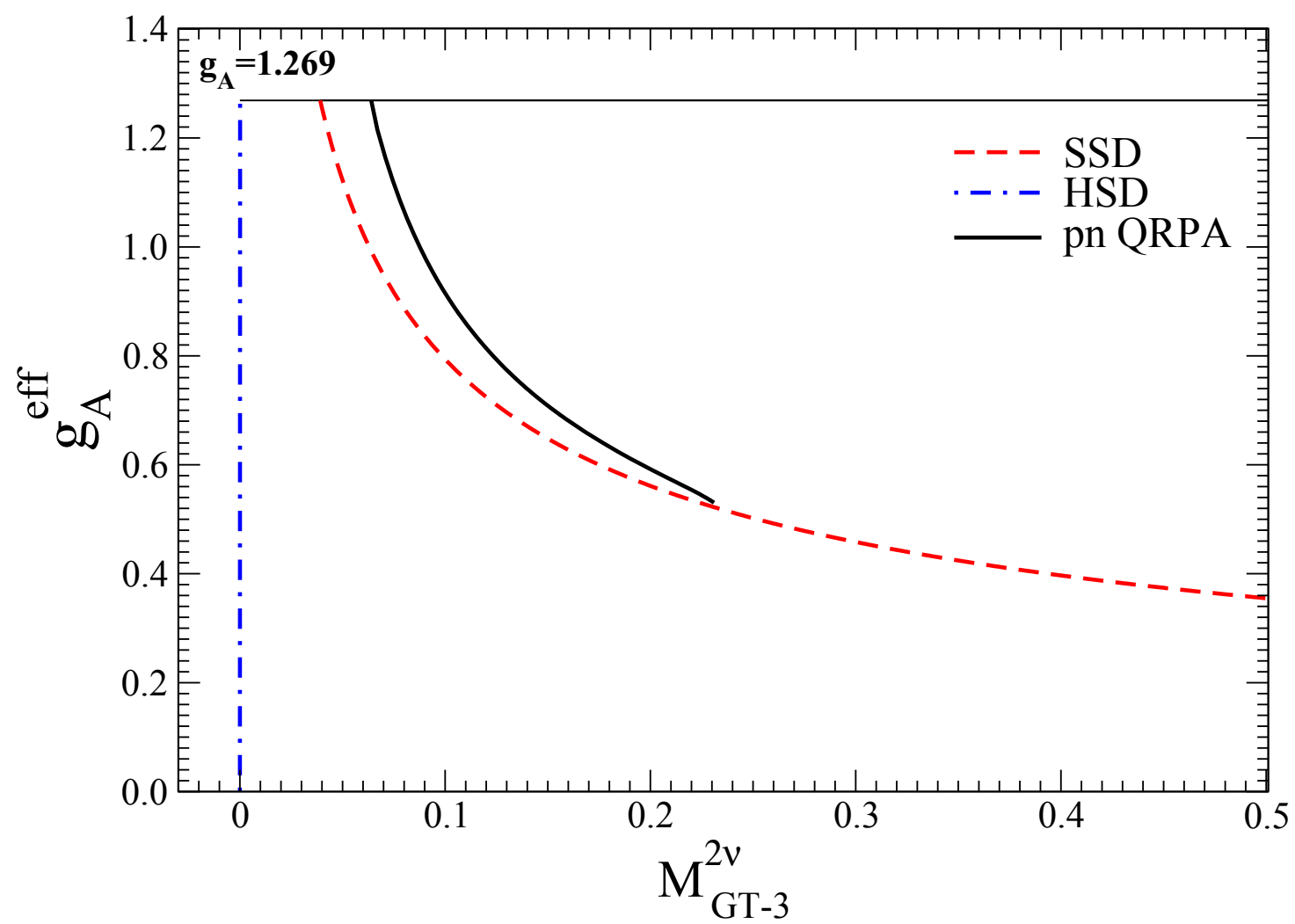

Figure 2. (Color online) The effective axial-vector coupling constant $g_{A}^{\mathrm{eff}}$ as function of the matrix element $M_{G T-3}^{2 v}$ for $2 v \beta \beta$-decay of ${ }^{100}$ Mo. The QRPA dependence is shown by solid line. The SSD and HSD predictions are presented by dashed and dot-dashed lines, respectively.

contains energy denominator to the third power, a role of transitions through higher lying states of the intermediate nucleus are expected to be negligible.

Within the QRPA with partial restoration of isospin symmetry [8] calculated running sums of matrix elements $M_{G T-1}^{2 v}$ and $M_{G T-3}^{2 v}$ as a function of the excitation energy $E_{e x}$ counted from the ground state are presented for the $2 v \beta \beta$-decay of ${ }^{76} \mathrm{Ge},{ }^{82} \mathrm{Se},{ }^{96} \mathrm{Zr}$ and ${ }^{100} \mathrm{Mo}$ in Fig. 1 . The unquenched value of axial-vector coupling constant is assumed $\left(g_{A}^{\mathrm{eff}}=g_{A}=1.269\right)$. Recall that in the QRPA treatment of double-beta decay processes a common practice is to fix parameters of nuclear Hamiltonian by reproducing the measured $2 v \beta \beta$-decay half-life for a given value of $g_{A}^{\text {eff }}$. From Fig. 1 we see that matrix element $M_{G T-3}^{2 v}$ is clearly determined by transitions through the lowest states of the intermediate nucleus unlike $M_{G T-1}^{2 v}$, which depends also on the transitions through higher lying states even from the region of Gamow-Teller resonance. We notice also a mutual cancellation among different contributions in the case of $M_{G T-1}^{2 v}$. Thus, it is expected that the calculation of $M_{G T-3}^{2 v}$ might be more reliable when compared with evaluation of $M_{G T-1}^{2 v}$, especially in nuclear models which describe accurately lowest excitations of a nucleus like the interacting shell model.
In Table 1, we present values of $M_{G T-1}^{2 v}$ and $M_{G T-3}^{2 v}$ for the $2 v \beta \beta$-decay of ${ }^{76} \mathrm{Ge},{ }^{82} \mathrm{Se},{ }^{96} \mathrm{Zr}$ and ${ }^{100} \mathrm{Mo}$, which were obtained within the QRPA with partial restoration of the isospin symmetry by assuming $g_{A}^{\text {eff }}=0.8,1.0$ and 1.269. The strengths of isocalar and isovector interactions of nuclear Hamiltonian were adjusted following the procedure in [8]. The kinematical phase-space factors $G_{0}^{2 v}$ and $G_{2}^{2 v}$ were determined by considering the exact Dirac wave functions with finite nuclear size and electron screening for emitted electrons [6]. The quantities $P_{0}^{2 v}$ and $P_{2}^{2 v}$ denote the leading and next to leading contributions of Taylor expansion in $\varepsilon$ to $2 v \beta \beta$-decay width relative to the full width, respectively. From four analyzed nuclei in Table 1 the largest value of $P_{2}^{2 v}$ is found for ${ }^{100} \mathrm{Mo}(\sim 20 \%)$ and the smallest value for ${ }^{76} \mathrm{Ge}(\sim 3 \%)$.

By introducing the ratio $\xi_{13}^{2 v}$ of nuclear matrix elements $M_{G T-3}^{2 v}$ and $M_{G T-1}^{2 v}$,

$$
\xi_{13}^{2 v}=\frac{M_{G T-3}^{2 v}}{M_{G T-1}^{2 v}},
$$

the $2 v \beta \beta$-decay half-life can be written in a form without explicit dependence on $M_{G T-1}^{2 v}$ as follows: 


$$
\left[T_{1 / 2}^{2 v \beta \beta}\right]^{-1}=\left(g_{A}^{\mathrm{eff}}\right)^{4}\left|M_{G T-3}^{2 v}\right|^{2} \frac{1}{\left|\xi_{13}^{2 v}\right|^{2}}\left(G_{0}^{2 v}+\xi_{13}^{2 v} G_{2}^{2 v}\right) .
$$

The expression (18) can be exploited to determine the value of $g_{A}^{\text {eff }}$, if $\xi_{13}^{2 v}$ is determined phenomenologically from the shape of measured energy distributions of emitted electrons in the $2 v \beta \beta$-decay and a reliable calculation of nuclear matrix element $M_{G T-3}^{2 v}$ is performed, e.g., within the Interacting Shell Model.

The NEMO3 experiment measured single and summed electron differential decay rates of the $2 v \beta \beta$-decay of ${ }^{100}$ Mo with very high statistics of about 1 million events [11]. It allowed to obtain valuable information about the Single State Dominance (SSD) and Higher State Dominance (HSD) hypotheses [12], which discuss the importance of contributions to the $2 v \beta \beta$-decay NME from transitions through low-lying (SSD hypothesis [13]) and higher lying (the region of the GT resonance) intermediate nuclear states $[14,15]$. For these two particular cases we have

$$
\begin{aligned}
\xi_{13}^{2 v}\left({ }^{100} \mathrm{Mo}\right) & =0.37(\mathrm{SSD}) \\
& =0.00(\mathrm{HSD}) .
\end{aligned}
$$

The real value of $\xi_{13}^{2 v}\left({ }^{100} \mathrm{Mo}\right)$ is a subject of experimental analysis within the NEMO3 collaboration. Its value can be any. The $2 v \beta \beta$ differential decay rate is given by

$$
\begin{array}{r}
\frac{d \Gamma^{2 v}}{d E_{e_{1}} d E_{e_{2}} d(\cos \theta)}=\frac{1}{2} \frac{m_{e}}{8 \pi^{7}}\left(G_{\beta} m_{e}^{2}\right)^{4}\left(g_{A}^{\mathrm{eff}}\right)^{4} \frac{1}{m_{e}^{11}} \times \\
\left(M_{G T-1}^{2 v}\right)^{2} F_{0}\left(Z_{f}, E_{e_{1}}\right) p_{e_{1}} E_{e_{1}} F_{0}\left(Z_{f}, E_{e_{2}}\right) p_{e_{2}} E_{e_{2}} \times \\
\int_{0}^{E_{i}-E_{f}-E_{e_{1}}-E_{e_{2}}} E_{v_{1}}^{2} E_{v_{2}}^{2}\left[\left(1+\xi_{31}^{2 v} \frac{\varepsilon_{K}^{2}+\varepsilon_{L}^{2}}{\left(2 m_{e}\right)^{2}}\right)+\right. \\
\left.\left(1+\xi_{31}^{2 v} \frac{\varepsilon_{K}^{2}+\varepsilon_{L}^{2}}{\left(2 m_{e}\right)^{2}}\right) \frac{p_{e_{1}} p_{e_{2}}}{E_{e_{1}} E_{e_{2}}}\right] d E_{v_{1}}
\end{array}
$$

In Fig. 2, the effective axial-vector coupling constant $g_{A}^{\text {eff }}$ calculated within the QRPA with partial restoration of isospin symmetry [8] is plotted as function of the matrix element $M_{G T-3}^{2 v}$ for $2 v \beta \beta$-decay of ${ }^{100}$ Mo. Recall that the isoscalar neutron-proton interaction of the nuclear Hamiltonian was adjusted to reproduce correctly the measured $2 v \beta \beta$-decay half-live of ${ }^{100} \mathrm{Mo}$ [4] for each $g_{A}^{\text {eff }}$. The allowed values of $M_{G T-3}^{2 v}$ are within the range $(0.064,0.231)$ determined mostly by the pairing properties of involved initial and final nuclei. The SSD and HSD results are displayed as well. The experimentally determined value of $\xi_{13}^{2 v}\left({ }^{100} \mathrm{Mo}\right)$ from the shape of the energy distribution might help to discriminate among considered scenarios and to conclude about $g_{A}^{\text {eff }}$ for $M_{G T-3}^{2 v}$ calculated within nuclear structure theory.

It is worth to note that a significant progress has been achieved in double beta decay experiments recently. The $2 v \beta \beta$-decay mode has been measured with high statistics in the GERDA $\left({ }^{76} \mathrm{Ge}\right)[16]$, CUORE $\left({ }^{130} \mathrm{Te}\right)$ [17], KamlandZEN ( $\left.{ }^{136} \mathrm{Xe}\right)[18]$, and EXO $\left({ }^{136} \mathrm{Xe}\right)[19]$ experiments. Further double beta decay experiments, which will contain significantly larger amount of double beta decay radioactive source, are in construction (SuperNEMO) or consideration (nEXO, Legend, etc.) [20]. These facts open new perspectives also for determination $g_{A}^{\text {eff }}$ via the measurement of $\xi_{13}^{2 v}$.

\section{Conclusions}

In summary, a more accurate expression for the $2 v \beta \beta$ decay half-life was derived by taking into account dependence of energy denominators on lepton energies and using of a Taylor expansion, which allows to factorize calculation of phase-space factors and nuclear matrix elements. It was found that the value of additional contribution to the $2 v \beta \beta$-decay width corresponds to $3-20 \%$ of the full width for $A=76,81,96$ and 100 nuclear systems. The revised expression for $2 v \beta \beta$-decay half-life includes in addition to the well-known nuclear matrix element $M_{G T-1}^{2 v}$ also the double Gamow-Teller matrix element $M_{G T-3}^{2 v}$ with energy denominator to the third power. This matrix element is governed by the transitions through the lowest states of the intermediate nucleus. It was proposed that the effective axial-vector coupling constant $g_{A}^{\text {eff }}$ can be determined from the measured $2 v \beta \beta$-decay half-life, if the ratio $\xi_{13}^{2 v}$ of nuclear matrix elements $M_{G T-3}^{2 v}$ and $M_{G T-1}^{2 v}$ is deduced phenomenologically from the shape of energy spectrum of emitted electrons in the $2 v \beta \beta$-decay and nuclear matrix element $M_{G T-3}^{2 v}$ is reliably calculated, e.g., within the interacting shell model. In this way one avoids the problem of the importance of transitions through the higher lying states of the intermediate nucleus.

This work is supported by the VEGA Grant Agency of the Slovak Republic under Contract No. 1/0922/16, by Slovak Research and Development Agency under Contract No. APVV-14-0524, RFBR Grant No. 16-02-01104, Underground laboratory LSM - Czech participation to European-level research infrastructue CZ.02.1.01/0.0/0.0/16 013/0001733.

\section{References}

[1] J.D. Vergados, H. Ejiri, and F. Šimkovic, Rept. Prog. Phys. 75, 106301 (2012)

[2] J. Engel and J. Menéndez, Rept. Prog. Phys. 80, 046301 (2017)

[3] J. Suhonen, Front. in Phys. 5, 55 (2017)

[4] A. Barabash, Nucl. Phys. A 935, 52 (2015)

[5] D. Štefánik, F. Šimkovic, and A. Faessler, Phys. Rev. C 91, 064311 (2012)

[6] J. Kotilla and F. Iachello, Phys. Rev. C 85, 034316 (2012)

[7] F. Šimkovic, R. Dvornický, D. Štefánik, and A. Faessler, Phys. Rev. C 97, 034315 (2018)

[8] F. Šimkovic, V. Rodin, A. Faessler, and P. Vogel, Phys. Rev. C 87, 045501 (2013)

[9] A. Faessler, G.L. Fogli, E. Lisi, V. Rodin, A.M. Rotunno, and F. Šimkovic, J. Phys. G: Nucl. Part. Phys. 35, 075104 (2008)

[10] J. Barea, J. Kotila, and F. Iachello, Phys. Rev. C 87, 014315 (2013) 
[11] The NEMO-3 Collab., R. Arnold, et al., Phys. Rev. D 92, 072011 (2015)

[12] The NEMO-3 Collab., R. Arnold, et al., JETP Lett. 80, 377 (2004)

[13] J. Abad, A. Morales, R. Nunez-Lagos and A. Pacheco, Ann. Fis. A 80, 9 (1984)

[14] F. Šimkovic, P. Domin, and S.V. Semenov, J. Phys. G: Nucl. Part. Phys. 27, 2233 (2001)

[15] P. Domin, S. Kovalenko, F. Šimkovic, and S.V. Semenov, Nucl. Phys. A 753, 337 (2005)
[16] The GERDA Collab., M. Agostini, et al., Nature 544, 47 (2017)

[17] The CUORE Collab., K. Alfonso, et al., Phys. Rev. Lett. 115, 102502 (2015)

[18] The KamLAND-Zen Collab., A. Gando, et al., Phys. Rev. C 85, 045504 (2012)

[19] The EXO Collab., N. Ackerman, et al., Phys. Rev. Lett. 107, 212501 (2011)

[20] A.S. Barabash, arXiv:1702.06340[nucl-ex]. 